\title{
Comunicación interna y gestión de bienestar y felicidad en la empresa española
}

\section{Internal communication and happiness and wellbeing management in Spanish companies}

\author{
Andrea Castro-Martínez; Pablo Díaz-Morilla
}

Cómo citar este artículo:

Castro-Martínez, Andrea; Díaz-Morilla, Pablo (2020). “Comunicación interna y gestión de bienestar y felicidad en la empresa española”. Profesional de la información, v. 29, n. 3, e290324.

https://doi.org/10.3145/epi.2020.may.24

Artículo recibido el 12-11-2019

Aceptación definitiva: 16-03-2020

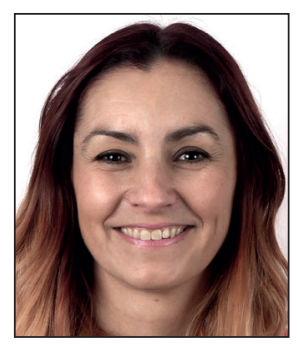

Andrea Castro-Martínez $\bowtie$

https://orcid.org/0000-0002-2775-625X

Universidad de Málaga

Facultad de Ciencias de la Comunicación. León Tolstoi, s/n. 29010 Málaga, España andreacastro@uma.es

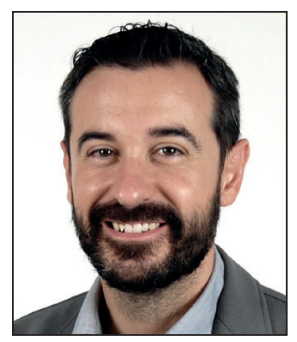

Pablo Díaz-Morilla https://orcid.org/0000-0002-7914-3391

EADE Estudios Universitarios Miguel Sel Gómez de la Cruz, 2. 29018 Málaga, España pablodmorilla@uma.es

\section{Resumen}

La gestión de la comunicación interna en las organizaciones ha experimentado un amplio desarrollo en los últimos años mediante el uso de diversas herramientas, muchas de ellas asociadas a los avances tecnológicos. Sin embargo, en muchas organizaciones continúa ligada a los departamentos de recursos humanos al entender que está vinculada a la relación con los trabajadores. En este contexto, caracterizado por la tendencia a fomentar la experiencia de los empleados para aumentar su implicación y posibilitar la gestión del cambio, se han introducido en las organizaciones los conceptos de felicidad y bienestar de la plantilla. Este trabajo profundiza en la relación de la comunicación interna y la felicidad y bienestar de los empleados a través de un cuestionario realizado a los responsables de comunicación interna de 82 empresas españolas que han sido reconocidas por sus acciones en comunicación interna por la consultora Great Place to Work y por el Observatorio de Comunicación Interna. Los resultados indican que la vinculación entre felicidad y comunicación interna es fuerte, pese a que el área de dirección de felicidad está muy poco implementada en las organizaciones españolas y a que en la mayoría de los casos depende del área de recursos humanos. En España el término más utilizado para designar este puesto es el de dirección de bienestar, seguido por el de dirección de felicidad y no tanto el término en inglés Chief happiness officer, como ocurre en otros países.

\section{Palabras clave}

Comunicación interna; Comunicación organizacional; Comunicación corporativa; Felicidad laboral; Bienestar laboral; Compromiso organizacional; Herramientas internas; Relaciones Públicas; Marketing interno; Organización; Director de felicidad; Dirección de felicidad; Dirección de bienestar; Chief happiness officer; España.

\begin{abstract}
The management of internal communication in organizations has undergone extensive development in recent years through the use of various tools, many of them associated with technological advances. However, in many organizations, it continues to be linked to human resources departments, based on the understanding that it is linked to the relationship with workers. In this context, characterized by the tendency to promote the experience of employees in order to increase their involvement and enable management change, the concepts of staff happiness and well-being have been introduced into organizations. This work delves into the relationship between internal communication and the happiness and well-being of employees using a questionnaire completed by internal communication managers in 82 Spanish companies that have been recognized for their actions in this area by the Great Place to Work consultancy firm and by the Internal Communication Observatory. The results indicate that the link between happiness and internal communication is strong, despite the fact that happiness management is implemented very little in Spanish organiza-
\end{abstract}


tions and in most cases is the responsibility of human resources. In Spain, the term most commonly used to designate this position is that of Wellbeing Director, followed by that of Happiness Director and less so Chief happiness officer, as occurs in other countries.

\section{Keywords}

Internal communication; Organizational communication; Corporate communication; Work happiness; Wellbeing at work; Organizational commitment; Internal tools; Public Relations; Internal marketing; Organization; Happiness director; Welfare director; Wellbeing director; Chief happiness officer; Spain.

\section{Introducción}

La concepción de que las Relaciones Públicas son una función vinculada a la dirección de las organizaciones y a sus aspectos gerenciales está ampliamente asentada (Grunig; Hunt, 2003; Cutlip; Center; Broom, 2001). También lo está desde la perspectiva de los stakeholders (Freeman; Reed, 1983) la importancia de los trabajadores como grupo de interés dentro de cualquier entidad. De este modo la comunicación interna $(\mathrm{Cl})$ tiene una gran relevancia en la comunicación corporativa (Ulloa-Tapia; Apolo-Buenaño; Villalobos-Arqueros, 2015) y actúa como elemento cohesionador dentro de la empresa (Arribas-Urrutia, 2000).

Los beneficios de la comunicación interna son intangibles y a largo plazo (Del Pozo-Lite, 2000) pero su buena gestión constituye también la base de una comunicación externa exitosa (Xifra; Lalueza, 2009). Así, un adecuado desarrollo de sus potencialidades permite a las organizaciones ser más competitivas a nivel interno y externo. La empresa actual, inserta en un entorno comunicativo que permite la retroalimentación constante y simultánea, se ve obligada a desarrollar una comunicación dinámica y en permanente evolución donde se pone de relevancia la participación de los públicos internos como agentes clave de este proceso, ya que su compromiso resulta decisivo para los objetivos de desarrollo de la organización y para convertirse en portavoces y embajadores de marca (Aguadero-Fernández, 2013). De este modo, las estrategias de $\mathrm{Cl}$ contribuyen a generar compromiso e implicación (Castro-Martínez; Díaz-Morilla, 2019). Esta vinculación emocional de los trabajadores con la organización a la que pertenecen se ve condicionada por la percepción sobre los niveles de bienestar y felicidad que la empresa les proporciona (Vasconcelos, 2008).

Esta investigación aborda la relación existente entre la gestión de la Cl y la gestión de la felicidad en empresas españolas reconocidas por sus buenas prácticas en $\mathrm{Cl}$ por la consultora Great Place to Work y por el Observatorio de Comunicación Interna. El objetivo general que se plantea es conocer el modo en que se establece dicha relación y la forma en que se ve plasmada en la estructura organizativa. Se fijan como objetivos secundarios los siguientes:

- Estudiar la configuración de los departamentos de comunicación interna de las empresas mencionadas.

- Establecer el uso que estas empresas hacen de los diferentes canales de comunicación interna.

- Identificar las tendencias en gestión de comunicación interna.

- Analizar el modo en que se estructura la gestión de la felicidad y el bienestar en las organizaciones españolas.

\section{Revisión bibliográfica}

Según señalan Grunig y Hunt (2003), aunque aún hay empresas que basan sus relaciones con los empleados en los modelos de promoción, información o en el bidireccional asimétrico, son muchas las que han avanzado en este sentido y han entrado en la era de la comunicación simétrica bidireccional aplicada al interior de la organización. Este planteamiento da relevancia a la comunicación interpersonal y al diálogo con la dirección, de especial importancia en las empresas menos estructuradas.

De este modo la $\mathrm{Cl}$, al potenciar la identificación del trabajador con la marca, está vinculada al marketing interno y al aumento de su motivación y productividad, al entender que el nuevo perfil de trabajador lo hace

"más crítico, más escéptico y, como consecuencia, más reacio a integrarse en instituciones que no le garanticen plenamente su desarrollo personal, social y económico" (Barranco, 1993, p. 52).

La $\mathrm{Cl}$ favorece así, además de la productividad, la retención de talento al potenciar la fidelización de los miembros de una organización y contribuir notoriamente a que se sientan partícipes de la cultura corporativa, se adhieran a ella y a sus valores de marca (Berceruelo, 2011). Los trabajadores necesitan estar informados para aumentar su nivel de compromiso (Vilanova-Giralt, 2016) por lo que la creación de corrientes de credibilidad y confianza es uno de los fines de la Cl (Barquero; Pérez-Senac; Barquero, 2010). Por su parte, Xifra (2011, pp. 129-130), que entiende que los trabajadores son el "público-motor" de la organización, señala cinco ejes para un desarrollo exitoso de la $\mathrm{Cl}$ :

- priorizar a las personas y la comunicación, ya que el clima psicológico de las organizaciones depende de la comunicación establecida y de cómo se responda a las expectativas de los empleados;

- comprender que objetivos sociales y económicos no son excluyentes, ya que la $\mathrm{Cl}$ contribuye a una mejor implementación de las decisiones que se adoptan en el seno de la organización;

- contar con el compromiso de la dirección, cuya implicación en los procesos comunicativos es necesaria;

- emplear las nuevas tecnologías de forma que faciliten la comunicación y que no interfieran en la motivación o sentimiento de pertenencia, sobre todo en los trabajadores que ejerzan sus tareas en remoto; 
- mantener una sinergia entre comunicación interna y externa, de modo que no se descuiden las relaciones internas en favor de proyectar hacia el exterior una determinada imagen.

Además, la $\mathrm{Cl}$

"es un agente de cambio, dado que apoya y facilita la introducción de nuevos valores o pautas de gestión, y la alineación de los empleados con los objetivos organizacionales" (Cuenca-Fontbona; Verazzi, 2018, p. 20).

A final de la década de 1990 la responsabilidad sobre la $\mathrm{Cl}$ en las empresas era muy variada y solía ser compartida, dependiendo de departamentos como recursos humanos o comunicación, pasando por marketing o personal, lo que dificultaba su gestión estratégica; además, en muchas ocasiones no recibía todo el apoyo necesario al ser vista como una responsabilidad añadida (Del-Pozo-Lite, 1997, pp. 153-154). Esta situación ha ido evolucionando, aunque aún existen muchas organizaciones en las que las Relaciones Públicas siguen sin concebirse como una función directiva y de toma de decisiones, por lo que su capacidad se ve mermada cuando ocupa una posición jerárquica inferior (Xifra, 2005). En la actualidad, dentro del organigrama empresarial es habitual en las grandes empresas que el área de comunicación integre de forma unificada la gestión de comunicación interna y externa, aunque en entidades de menor tamaño es común que la función de comunicación interna se sitúe en dependencia del área de recursos humanos (Rojas-Orduña, 2012; Aced, 2013). Hay autores que defienden que, pese a que comunicación interna y recursos humanos $(\mathrm{RRHH})$ deben trabajar de forma coordinada, es el departamento de comunicación quien debe gestionar toda la comunicación de manera unificada, incluyendo así la que se realiza hacia dentro de la organización (Almansa-Martínez, 2005).

Aunque predomina la vinculación al departamento de comunicación, la dependencia del área de $\mathrm{Cl}$ del departamento de RRHH sigue siendo muy importante

Los profesionales que se dedican a $\mathrm{Cl}$ en las empresas españolas se caracterizan por su alta cualificación, al tiempo que estos departamentos están adquiriendo una identidad propia dentro del organigrama empresarial (Miquel; Aced, 2019). Las nuevas necesidades a las que se enfrentan las empresas requieren de un grupo de profesionales interdisciplinar para la gestión estratégica de la comunicación (Carrillo-Durán, 2016).

Por otra parte, son muchas las herramientas de $\mathrm{Cl}$ que pueden emplearse (Andrade, 2005; Castillo-Esparcia, 2010; Almansa-Martínez, 2011; Cebrián-Herreros, 2012; De-las-Heras-Pedrosa; Ruiz-Mora; Paniagua-Rojano, 2018) y a las que se han sumado en los últimos años algunas que tienen su base en los avances tecnológicos. Sin embargo, el uso de diversos canales ofrece una mayor posibilidad de éxito ya que un programa eficaz de comunicación interna debe contemplar que los empleados demandan una

"comunicación sofisticada de alta tecnología, pero también un contacto personal con sus directivos" (Argenti, 2014, p. 402).

En general las estrategias de comunicación interna se enfocan en potenciar la motivación y el orgullo de pertenencia de los empleados, de forma que contribuyan al crecimiento de la entidad (García-Uceda, 2016). De este modo, la transmisión de activos intangibles constituye uno de los retos a los que se enfrentan las empresas (Barbeito-Veloso; Perona-Páez, 2019).

En este contexto, la gestión del bienestar y la felicidad se ha convertido en un área de interés creciente a nivel corporativo (Robertson; Cooper, 2001; Warr, 2013). Por ejemplo, un reciente estudio desarrollado en Francia (IFOP, 2020) indica que para el $82 \%$ de los encuestados una empresa es responsable de la felicidad de sus empleados; además, el $77 \%$ de los participantes opina que el funcionamiento organizativo de las empresas debe ser similar al de una democracia real, involucrando a los trabajadores en las decisiones estratégicas.

La satisfacción, el bienestar y la felicidad en el entorno laboral se han abordado desde diferentes perspectivas, como pueden ser la economía (Bauer, 2017), la psicología (Masrouki, 2019; Boniwell; Chabanne, 2017), la sociología del trabajo (Frayne, 2018; Barel; Frémeaux, 2016), los recursos humanos y el marketing (Fall; Safy-Godineau; Carassus, 2018; Delassus, 2019; Frey, 2018; Feuvrier, 2014) o la que vincula el nivel de bienestar laboral con la salud integral de los asalariados (Guillemin, 2018).

Por su parte, la comunicación interna también contribuye a mejorar estos aspectos de felicidad y bienestar a través del fomento de la cultura organizacional y del compromiso y fidelización de los empleados. Los índices más altos de implantación de cultura organizacional se relacionan con mayores niveles de felicidad percibida por los integrantes de una entidad (Álvarez-Nobell; Muñiz, 2013). Las características de una organización son importantes en cuanto a la posibilidad de establecer el diálogo dentro de la misma, así como su cultura interna, que ha de ser compatible con él (Huang; Yang, 2015) y que está relacionada con la eficiencia (Díaz-Soloaga, 2019). Los estilos de comunicación gerencial tienen impacto en las actitudes y comportamientos de los trabajadores (Dasgupta; Suar; Singh, 2013) y la Cl facilita una relación favorable entre éstos y la organización (Karanges et al., 2015). Fomentar una cultura de vitalidad y de aprendizaje contribuye a que los empleados prosperen y estén más satisfechos, lo que se puede lograr con acciones como facilitar la autonomía y toma de decisiones, compartir información, reducir los comportamientos incívicos y aportar información sobre los niveles de desempeño (Spreitzer; Porath, 2016).

No existe una definición científica asentada del concepto de felicidad en el trabajo porque cada entidad posee su propio sistema organizativo, normas y valores, lo que hace que sea preferible considerar la felicidad en el trabajo 
"como la calidad emergente del sistema de interacciones resultante de la calidad de las corrientes de comunicación e información dentro de la organización” (Hassani, 2017).

En este sentido y ante los crecientes casos de burn-out y de sufrimiento laboral, muchas empresas han optado por implementar canales de comunicación ascendentes para reforzar el bienestar de los empleados en su lugar de trabajo principalmente a través de dos vías: la creación de una figura responsable de la gestión del bienestar y mediante el diseño de espacios de convivencia y relajación (D’Almeida; Libaert, 2018). La figura encargada de unificar la gestión del bienestar y la felicidad es el Chief happiness officer ( $\mathrm{CHO}$ ), responsable de felicidad o responsable de calidad de vida en el trabajo, y que algunas organizaciones ya han comenzado a implementar (Bardon; Josserand, 2018; Vanhée, 2013). Surgida en empresas de Silicon Valley, esta responsabilidad puede estar asociada a la dirección de $\mathrm{RRHH}$ o a la de $\mathrm{Cl}$ y consiste en crear eventos que promuevan una sensación de bienestar entre los empleados (D’Almeida; Libaert, 2018).

El puesto de responsable de felicidad no está aún claramente consolidado, pero su función aúna trabajo de comunicación y de diagnóstico y vigilancia del clima social de la entidad y emplea indicadores como la calidad de la comunicación y las relaciones en la empresa, la fidelización, compromiso y motivación o la tasa de absentismo (Beau, 2019). De este modo pretende dar respuesta a las expectativas de los empleados al tiempo que ejerce de intermediario entre la plantilla, la dirección y el departamento de RRHH (Beau, 2019). Se trata de una profesión innovadora que surge en organizaciones que pretenden aumentar la retención y la productividad de sus empleados a través de la creación de un ambiente de trabajo agradable y que se sitúa

"a medio camino entre la comunicación y la gestión de los recursos humanos y que también incluye trabajar en cuestiones estratégicas de la empresa" (Najeh, 2019, p. 21).

Por todo lo expuesto, es conveniente analizar la vinculación entre $\mathrm{Cl}$ y felicidad para conocer si la gestión de la primera puede beneficiar al bienestar y satisfacción dentro de la organización.

\section{Metodología}

Esta investigación se basa en un diseño metodológico mixto que a través de un cuestionario plantea preguntas de tipo cuantitativo y cualitativo para enriquecer el conocimiento del fenómeno estudiado (Wimmer; Dominick, 1996). El cuestionario, que ha sido suministrado por vía digital y mediante archivo autocompletable por cuestiones de filtros de seguridad de las entidades, se compone de 28 preguntas de respuestas dicotómicas, múltiples y abiertas, estructuradas en cinco bloques (tabla 1).

Tabla 1. Relación entre bloques del cuestionario y objetivos de la investigación.

\begin{tabular}{|l|l|}
\hline \multicolumn{1}{|c|}{ Bloque del cuestionario } & \multicolumn{1}{c|}{ Objetivo } \\
\hline Organización del departamento de Cl de la empresa. & $\begin{array}{l}\text { Estudiar la configuración de los departamentos de Cl de las empresas mencio- } \\
\text { nadas. }\end{array}$ \\
\hline Uso de la Cl en la entidad. & Establecer el uso que estas empresas hacen de los sistemas de Cl. \\
\hline Valoración de herramientas y tendencias en el uso de la Cl. & Identificar las tendencias en gestión de comunicación interna. \\
\hline $\begin{array}{l}\text { Relación entre Cl y felicidad. } \\
\text { Dirección de felicidad en la empresa. }\end{array}$ & $\begin{array}{l}\text { Analizar el modo en que se estructura la gestión de la felicidad y el bienestar en } \\
\text { las organizaciones españolas. }\end{array}$ \\
\hline
\end{tabular}

La muestra está compuesta por las marcas que han formado parte entre 2014 y 2018 del ranking Great Place to Work y de los Premios del Observatorio de Comunicación Interna ya que son empresas que han visto reconocidas de manera previa sus buenas prácticas en $\mathrm{Cl}$ por parte de estas dos entidades, relevantes en el ámbito académico y profesional (Medina, 2012; Ramilo-Méndez, 2012). Inicialmente la muestra estaba compuesta por 141 empresas, de las que 4 se repiten entre los dos grupos y 6 han desaparecido o se han integrado en otras marcas. Ha habido 11 empresas que han rechazado colaborar por motivos de confidencialidad. Finalmente 82 organizaciones han participado en el cuestionario a través de las respuestas aportadas por sus responsables de comunicación interna.

\section{Resultados}

\subsection{Organización y gestión de $\mathrm{Cl}$}

Las empresas encuestadas pertenecen a diversos sectores, entre los que destacan con mayor representación Banca/Financiero/ Seguros (18,3\%), Tecnología (11\%), Retail/Textil (7,3\%), Alimentación (6,1\%), Industria/Química/Reciclaje $(6,1 \%)$ y Transporte $(4,9 \%)$. Por tamaño de la plantilla predominan las empresas que superan los 1.000 empleados $(45,1 \%)$, seguidas por las del 251 -

Tabla 2. Departamentos responsables de la gestión de la $\mathrm{Cl}$.

\begin{tabular}{|l|c|}
\hline Departamento responsable & $\%$ \\
\hline Comunicación & 46,3 \\
\hline Recursos humanos & 36,5 \\
\hline Comunicación + RRHH & 7,6 \\
\hline Marketing & 2,4 \\
\hline Dirección general/CEO & 2,4 \\
\hline Oficina de comunicaciones estratégicas & 2,4 \\
\hline Vicepresidencia de relaciones estratégicas & 1,2 \\
\hline Sin departamento específico & 1,2 \\
\hline
\end{tabular}




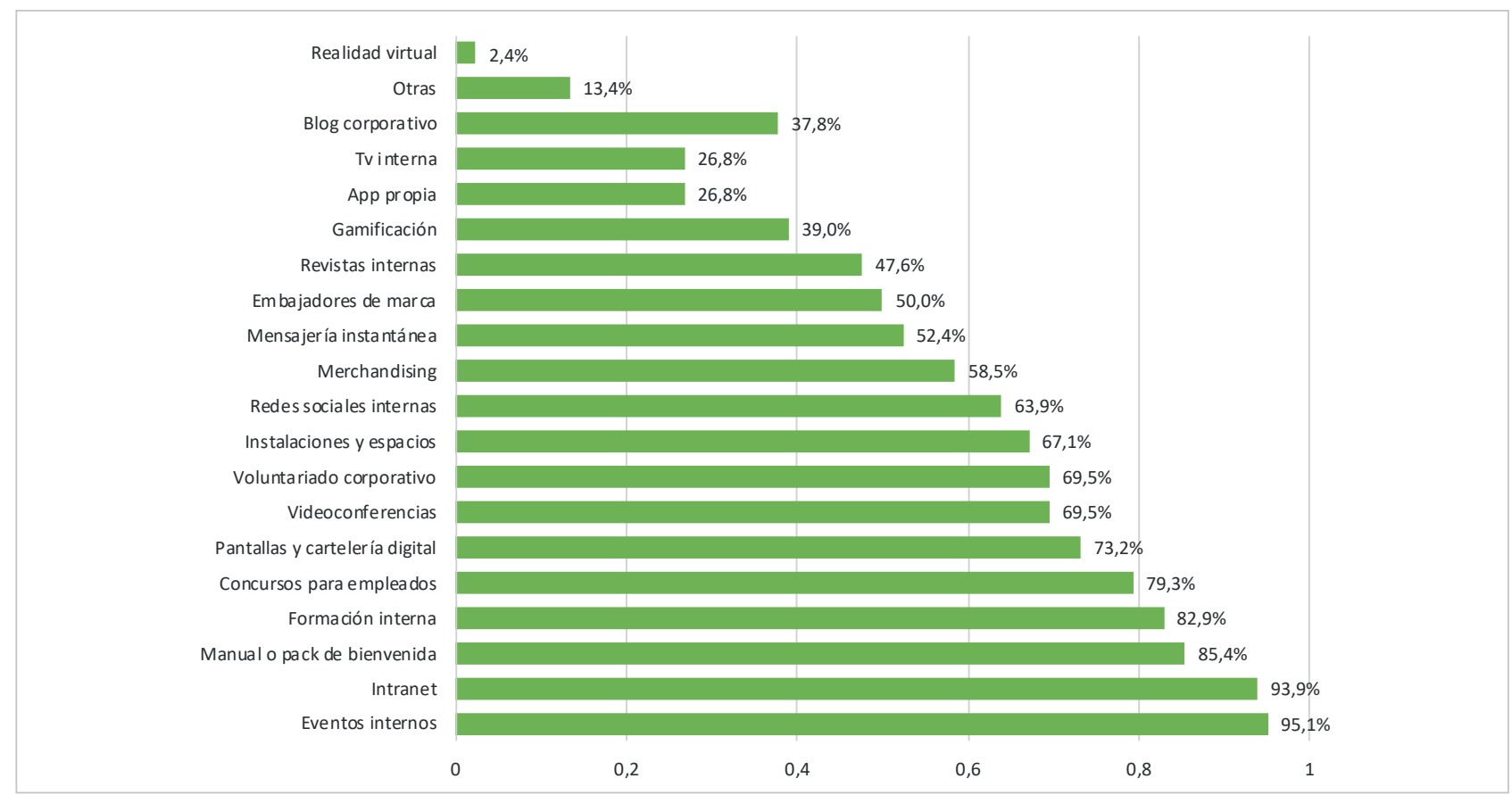

Gráfico 1. Medios de $\mathrm{Cl}$ empleados en las organizaciones.

500 empleados (20,7\%), las de 501-1.000 empleados (12,2\%), 101-200 empleados (12,2\%), de 50 a 100 empleados $(7,3 \%)$ y las de menos de 50 empleados $(2,4 \%)$.

En cuanto a la gestión de la $\mathrm{Cl}$, ésta depende mayoritariamente de los departamentos de comunicación (46,3\%) seguidos por $\mathrm{RRHH}$, que goza de gran importancia (tabla 2).

La media de empleados destinados a comunicación interna es de 4, siendo lo más habitual contar con dos trabajadores $(27,5 \%)$ y contabilizando sólo 6 departamentos con 10 o más empleados. En su composición predominan las mujeres $(71,8 \%)$ frente a los hombres (28,2\%). El nivel formativo es de grado/licenciatura o superior en el $81,9 \%$ de los casos, aunque apenas un 4,7\% cuenta con un doctorado. La titulación cursada mayoritariamente por los empleados dedicados a $\mathrm{Cl}$ es comunicación, aunque también están presentes otras como recursos humanos, económicas/empresariales, marketing, derecho, psicología o sociología.

En la estrategia de $\mathrm{Cl}$ lo más habitual es que se cuente con un plan diseñado y planificado previamente (83\%), frente a las acciones puntuales y esporádicas (11\%), el mix de ambos (3,6\%) o la espontaneidad en los mensajes $(1,2 \%)$. Por su parte, los medios de $\mathrm{Cl}$ que más se emplean en las organizaciones (gráfico 1) son eventos internos e intranet.

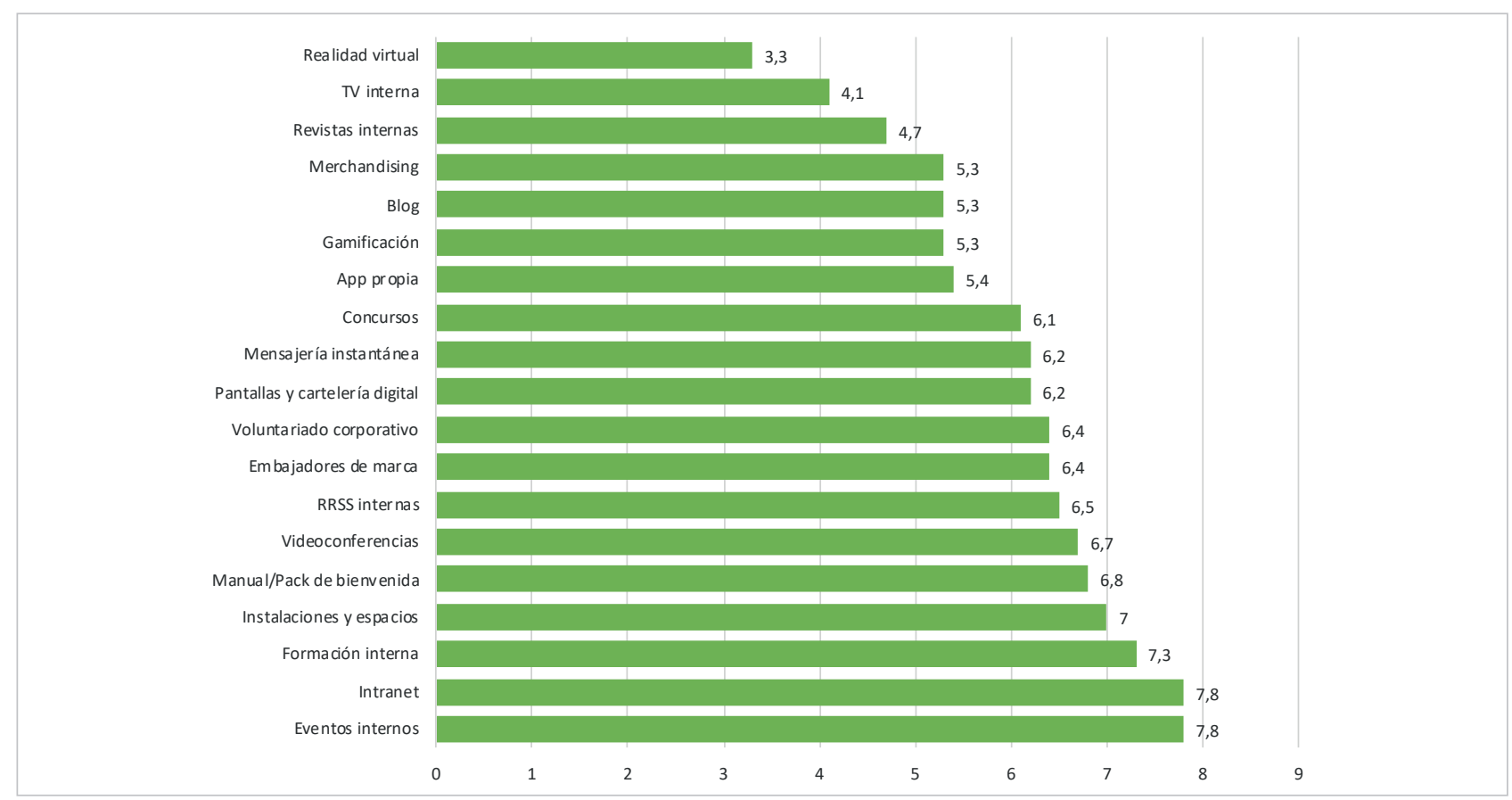

Gráfico 2. Valoración de las herramientas de $\mathrm{Cl}$ (sobre 10). 
En cuanto al futuro de la $\mathrm{Cl}$, un $84,1 \%$ indica que pasa por emplear un mix de herramientas digitales combinadas con relaciones interpersonales con tendencias vinculadas a la participación e interactividad, la integración, la gamificación y la digitalización, como el uso de redes sociales, aplicaciones internas o realidad virtual. En este contexto destaca el uso del móvil y el desarrollo de comunicaciones personales.

Los medios de $\mathrm{Cl}$ más valorados (gráfico 2) son eventos internos, intranet, formación interna, instalaciones y espacios que fomenten la comunicación y el manual/pack de bienvenida, algunas de las cuales coinciden con las más empleadas por las organizaciones estudiadas.

\subsection{Comunicación interna, dirección de felicidad y bienestar}

Prácticamente en todas las organizaciones estudiadas $(96,3 \%)$ se desarrollan acciones para potenciar el bienestar de la plantilla. Las medidas adoptadas en este sentido son muy diversas:

- planes vinculados a la mejora de la salud y la seguridad en el seno de la organización: Empresa Saludable, Plan salud \& bienestar, Programa t+Vida, Semana de la seguridad y la salud, Semana de la salud y la diversidad, deshabituación tabáquica, consumo de fruta...

- medidas para potenciar el bienestar físico y emocional: fisioterapia, taller de espalda, relajación, coaching, meditación, medidas de desarrollo y crecimiento personal, mindfulness...

- programas de nutrición y fomento del deporte y hábitos saludables: Programa Wellness, BeHealthy, nutricionista, gimnasio, clases de yoga, club de corredores, defensa personal...

- eventos culturales y de convivencia: celebraciones, Family day, visitas a museos, voluntariado, desayunos con directivos...

- rediseño de espacios de trabajo que fomenten la comunicación y el bienestar: más luminosos, de concepto abierto, mejor temperatura, duchas en las instalaciones...

- medidas de conciliación y felicidad laboral: flexibilidad horaria y espacial, intercambio de turnos, teletrabajo, jornada reducida algunos días, semana de cuatro días laborables...

- políticas de compensación y beneficios: seguros sociales, cheque gourmet, cheque guardería, abono transporte, ayudas económicas, tarjetas descuento, asesoramiento legal y psicológico en materia de violencia machista...

- encuestas de clima, entrevistas y seguimiento personalizado del bienestar y el desempeño, estudios de riesgos psicosociales, campañas de mejora de la experiencia del empleado...

Muchas de estas acciones coinciden con canales de comunicación interna ya implementados y los programas y medidas suelen contar con campañas propias de comunicación a nivel interno para darlas a conocer entre los asalariados.

Los motivos para perseguir la felicidad por parte de las organizaciones son los siguientes:

- planteamientos éticos relacionados con la condición de los empleados como seres humanos (38,6\%);

- un aumento de la productividad y el beneficio empresarial $(30,1 \%)$;

- una mezcla de los dos anteriores (14,5\%);

- motivar a los empleados para fomentar su alineación con los objetivos de negocio (7,2\%);

- fidelización, retención del talento y mejora de la reputación de la entidad (6\%);

- otros motivos (3,6\%).

Para el 96,3\% de la muestra es muy evidente la vinculación entre la gestión de la $\mathrm{Cl}$ y el nivel de felicidad, satisfacción y bienestar de la plantilla de una organización. Todos los encuestados creen que la $\mathrm{Cl}$ puede influir positivamente en la felicidad de los trabajadores y más del $80 \%$ le otorga una influencia de entre 8 y 10 puntos en una escala sobre 10 (gráfico 3).

Los aspectos en los que la $\mathrm{Cl}$ puede incidir en la felicidad de la plantilla (gráfico 4) son el aumento de motivación, la identificación con la marca, la fidelización, la mejora del desempeño,

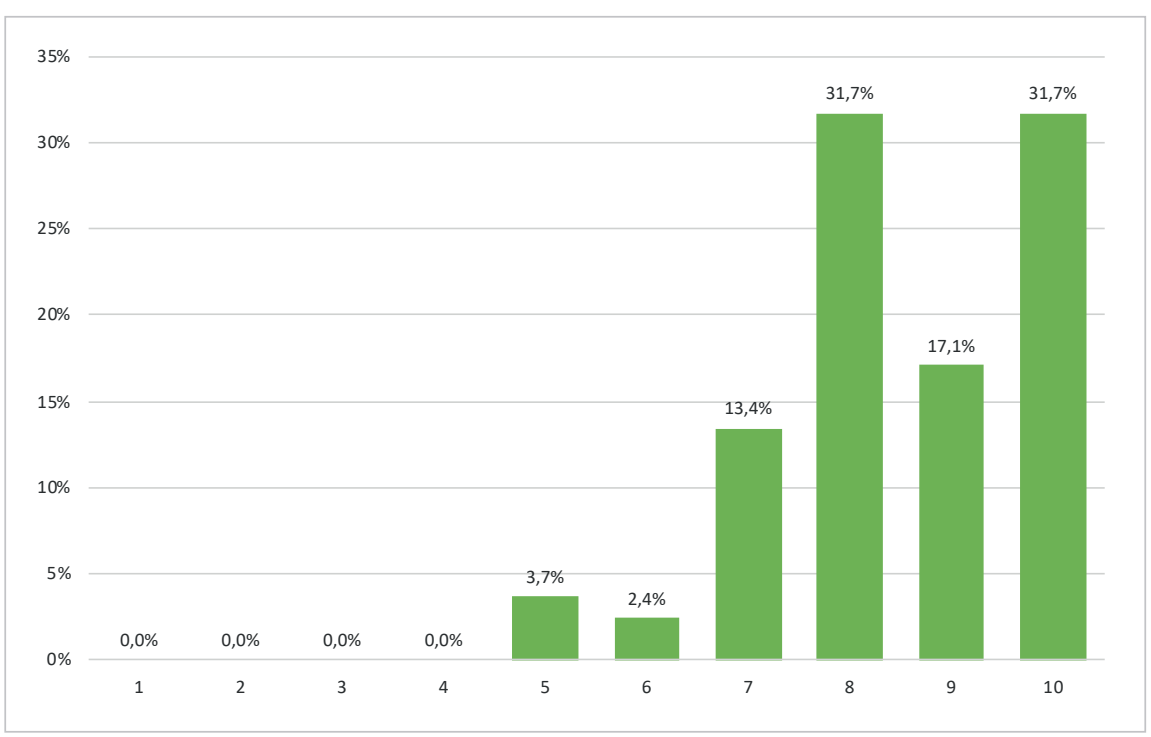

Gráfico 3. Medida en la que la $\mathrm{Cl}$ puede influir en la felicidad de la plantilla (sobre 10 puntos). 
la productividad, la mejora de la imagen de la entidad ante públicos internos y externos y la captación de prescriptores.

La dirección de Felicidad se trata de una figura conocida para el $70,7 \%$ de la muestra y desconocida para el $29,3 \%$. El término con el que la identifican (gráfico 5) es mayoritariamente dirección de Bienestar seguido por dirección de Felicidad y Chief happiness officer. Otros términos empleados para referirse a este puesto son Embajadores de la felicidad, Engagement manager, dirección de Personas y Bienestar o responsable de Experiencia del empleado. Cabe resaltar que un $28 \%$ no conocía ningún término para designar esta función.

Sobre la necesidad de que esta figura exista en las organizaciones, el $35,4 \%$ se muestra a favor y el $50 \%$ indica que tal vez sea necesaria. Hay un $14,6 \%$ que no cree que sea un puesto necesario, principalmente porque sus funciones ya se desarrollan en la actualidad a cargo de otros departamentos.

En cuanto al área de la organización a la que creen que debe pertenecer la dirección de Felicidad predomina RRHH (51,2\%), seguida por una mezcla entre comunicación y RRHH $(31,7 \%)$, comunicación (4,9\%) y otras áreas (6\%) como la Dirección general o la Dirección de responsabilidad social. Un $8,5 \%$ cree que debe ser un departamento independiente. Por su parte, entre las responsabilidades que según los encuestados corresponden a la dirección de Felicidad (tabla 3) se incluyen:

- la mejora del entorno y el clima laboral,

- potenciar el salario emocional,

- aumentar la motivación,

- retener el talento,

- ejercer la escucha activa,

- aumentar la implicación, y

- potenciar la $\mathrm{Cl}$, entre otras.

El que responsabilidades como potenciar la $\mathrm{Cl}$ y la escucha activa estén asociadas a este puesto evidencia que la $\mathrm{Cl}$ no es simplemente un factor que ayuda a gestionar la satisfacción, sino que conforma la felicidad y el bienestar dentro de una organización.

La implementación de la dirección de Felicidad en la empresa española es muy escasa, opinando la mayoría de la muestra $(65,8 \%)$ que en el contexto nacional se emplea nada o muy poco (gráfico 6). Por su parte, el $26,8 \%$ señala que esta figura ya está presente en la empresa española.
Tabla 3. Responsabilidades de la dirección de Felicidad.

\begin{tabular}{|l|c|}
\hline \multicolumn{1}{|c|}{ Responsabilidades } & $\begin{array}{c}\text { Relevancia } \\
\text { sobre 5 puntos }\end{array}$ \\
\hline Mejorar el entorno y el clima laboral & 4,8 \\
\hline Potenciar el salario emocional & 4,7 \\
\hline Aumentar la motivación & 4,6 \\
\hline Retener el talento & 4,4 \\
\hline Escucha activa & 4,4 \\
\hline Aumentar la implicación & 4,3 \\
\hline Mejorar la imagen de la organización entre sus empleados & 4,3 \\
\hline Potenciar la racionalización de horarios y la conciliación laboral & 4,2 \\
\hline Potenciar la Cl & 4,1 \\
\hline Reconocer la valía de los empleados & 4,0 \\
\hline Asesorar a la Dirección & 4,0 \\
\hline Otras & 2,7 \\
\hline
\end{tabular}


La mayoría de las organizaciones estudiadas gestionan la felicidad de manera informal $(52,4 \%)$ frente a un $29,3 \%$ que la gestiona de modo formal a través de estructuras organizativas asociadas a ella. El $18,3 \%$ no contempla ni trabaja la gestión de la felicidad. De entre el 29,3\% de organizaciones que gestionan la felicidad y el bienestar de manera formal y estructurada, tan sólo en el $11 \%$ de los casos existe la figura del director de Felicidad. No hay un perfil único de empresa que implemente este puesto: ni en relación con los sectores productivos, ya que todas pertenecen a áreas diferentes; ni por su tamaño, pues tiene presencia en empresas de 101 a 250 empleados (11,1\%), de 251 a 500 (22,2\%), de 501 a 1000 (22,2\%) y de más de 1.000 trabajadores (44,4\%). Sí se aprecia que la dirección de Felicidad o Bienestar es algo más común en empresas de mayor tamaño. El 89\% de los encuestados en cuya empresa existe este rol lo consideran necesario pese a que indican que el nivel de implementación de la figura a nivel general en la empresa española es de 4 puntos sobre 10.

Esta figura, en las empresas en las que tiene presencia, está vinculada al área de recursos humanos (63,3\%), o constituye una responsabilidad compartida entre comunicación y RRHH (13,3\%). En algunas organizaciones está vinculada a otras responsabilidades como Dirección general, Dirección de bienestar social o Dirección de gestión social y corporativa y sólo en una organización se trata de un departamento independiente. Todos los encuestados en cuya empresa existe la dirección de Felicidad indican que debe pertenecer al área en la que actualmente se inscribe, por lo que parecen estar conformes con su situación en el organigrama.

Las funciones que desempeña la dirección de Felicidad en las entidades que disponen de ella se resumen en las siguientes:

- Medir la felicidad y proporcionar bienestar a toda la organización.

- Aumentar la motivación.

- Retener el talento.

- Desarrollar estrategias que refuercen el bienestar físico y emocional mediante programas de comportamiento, cuidado de los empleados o salud y seguridad.

- Organizar actividades que fomenten el bienestar y la felicidad general de la comunidad como reuniones con 'Embajadores de la felicidad' a través de happiness groups, creación de grupos de comunicación y cultura corporativa.

- Emplear los sistemas de comunicación interna para transmitir los valores de la marca, su misión, propósito y objetivo de negocio o fomentar la confianza y el respeto entre todos los miembros de la organización.

En algunos casos la dirección de Felicidad se responsabiliza también de todas las iniciativas relacionadas con la captación, desarrollo y fidelización de talento, $\mathrm{Cl}$, evaluación de empleados, evaluación organizacional, cultura corporativa, conciliación, igualdad, etc.

Cabe destacar que hay empresas que, aunque no disponen de dirección de Felicidad propiamente dicha, sí desarrollan sus funciones a través de otras figuras con distintas denominaciones como Responsable de experiencia de empleado (departamento de Talento-RRHH), Compensación \& beneficios y Gestión del talento (RRHH), Unidad de desarrollo profesional, Experiencia de empleado y cultura, Dirección de gestión social y corporativa, Health and safety manager o Clima.

\section{Conclusiones y discusión}

Este trabajo alcanza su objetivo general al reflejar el modo en que se establece la relación entre la gestión de la comunicación interna $(\mathrm{Cl})$ y la gestión de la felicidad de las empresas españolas reconocidas por sus buenas prácticas en $\mathrm{Cl}$ por la consultora Great Place to Work y por el Observatorio de Comunicación Interna, y la forma en que dicha relación se refleja en su estructura organizativa. Prácticamente la totalidad de los representantes de las empresas estudiadas se muestra de acuerdo en que existe un vínculo entre la gestión de la $\mathrm{Cl}$ y el nivel de felicidad, satisfacción y bienestar que puede alcanzar la plantilla de una entidad. Como indican algunos autores, esto se debe a que la $\mathrm{Cl}$ tiene la capacidad de influir de manera positiva en estos aspectos a través del fomento del diálogo (Huang; Yang, 2015), el compromiso y la implicación 
(Castro-Martínez; Díaz-Morilla, 2019), el aumento de la eficiencia (Díaz-Soloaga, 2019) y la productividad (Barranco, 1993), el establecimiento de relaciones positivas entre la organización y sus miembros (Karanges et al., 2015) y la alineación de los asalariados con los objetivos de la marca (Cuenca; Verazzi, 2018). Todas las empresas analizadas desarrollan acciones para promover el bienestar y la felicidad de sus empleados y muchas de estas medidas coinciden con herramientas de comunicación interna como canales de comunicación o eventos. Además, un aspecto clave para implementarlas son las campañas de $\mathrm{Cl}$ con las que estas acciones se promueven entre la plantilla. A nivel organizativo son muchas las empresas en las que la gestión de la satisfacción, el bienestar y la felicidad de los empleados se vincula a las responsabilidades de $\mathrm{Cl}$. Esto puede suceder bien en dependencia del departamento de comunicación o bien a través del de recursos humanos, que en muchos casos integra el ámbito de comunicación interna de la entidad.

Los objetivos secundarios planteados por este trabajo también se alcanzan de manera íntegra. El primero de ellos, referido al estudio de la configuración de los departamentos de comunicación interna arroja unos resultados que coinciden con otras investigaciones previas (Carrillo-Durán, 2016; Miquel; Aced, 2019) al constatar el alto nivel formativo y la multidisciplinariedad de los profesionales dedicados a la comunicación interna.

El segundo objetivo secundario aborda el uso de los medios de $\mathrm{Cl}$. Los resultados de este trabajo muestran de forma detallada las diferentes herramientas que se utilizan en este ámbito, así como las más empleadas y las mejor valoradas dentro de las organizaciones, que en su mayoría trabajan a partir de un plan estratégico previamente diseñado. Estos datos vienen a reforzar los planteamientos de otros autores que señalan tanto la variedad de sistemas de $\mathrm{Cl}$ de las que las empresas disponen en la actualidad como la importancia de trabajar con ellas dentro de una estrategia de comunicación planificada (Cebrián-Herreros, 2012; De las Heras-Pedrosa; Ruiz-Mora; Paniagua-Rojano, 2018).

El tercer objetivo secundario se centra en identificar las tendencias en gestión de comunicación interna. Para los responsables de $\mathrm{Cl}$ de las entidades analizadas el futuro se verá condicionado por la integración de instrumentos digitales, especialmente de dispositivos móviles, y de comunicaciones personales, que permiten un trato más cercano y directo hacia los públicos internos. Estas tendencias coinciden con las ya indicadas por autores como Argenti (2014).

El último objetivo secundario consiste en analizar el modo en que se estructura la gestión de la felicidad y el bienestar en las organizaciones españolas. Mayoritariamente esta gestión se realiza de manera informal, sin una presencia fuerte ni netamente definida de estructuras orgánicas que se asocien a ella de manera unificada. También es importante el número de empresas que no trabajan y ni siquiera contemplan la gestión del bienestar y la felicidad de sus asalariados, al menos de manera directa. Dentro de las entidades que de forma estructurada abogan por el fomento y cuidado de estos temas, se aprecia una gran diversidad de posibilidades organizativas que abarcan desde la dependencia del área de $\mathrm{Cl}$ al de RRHH o a la Dirección general, pasando por otras estructuras centradas en el cuidado del empleado. La figura del director de Felicidad es muy escasa, ya que únicamente está presente en el $11 \%$ de las empresas estudiadas. No se identifica un perfil de empresa en el que esta función tenga presencia, aunque resulta más habitual en entidades que superan los 1.000 empleados. El área a la que se asocia mayoritariamente es la de RRHH, aunque también se muestra como una responsabilidad compartida entre comunicación y recursos humanos e incluso hay empresas que tienen otras estructuras jerárquicas. Esto coincide en parte con lo señalado por autores como D’Almeida y Libaert (2018) o Najeh (2019) que identifican las áreas de RRHH y comunicación como las más habituales para la dirección de Felicidad. Pese a la poca implementación en España de esta función, la mayoría de responsables la conoce y opta por denominarla dirección de Bienestar o dirección de Felicidad en lugar del término Chief happiness officer, más habitual en otros países (Bardon; Josserand, 2018; Vanhée, 2013).

Los objetivos de este puesto son no sólo mejorar el entorno, el clima laboral o la motivación y fidelización, sino también en muchos casos realizar funciones de comunicación, en coincidencia con lo señalado por otros autores (Beau, 2019; Hassani, 2017; Porath; Spreitzer, 2016; Álvarez-Nobell; Muñiz, 2013). De este modo la escucha activa y el potenciar la $\mathrm{Cl}$ son elementos que forman parte de la gestión de la felicidad en las organizaciones.

El papel de la $\mathrm{Cl}$ interna resulta muy relevante para el fomento de la satisfacción y la felicidad en el seno de las organizaciones, aunque en numerosas ocasiones sea el área de RRHH la encargada directa de su gestión. Debido a que la vinculación entre $\mathrm{Cl}$ y felicidad es sólida y probablemente continúe su desarrollo en el futuro, es recomendable que la gestión de la dirección de la felicidad y el bienestar en las organizaciones se trabaje de forma coordinada entre el departamento de comunicación y el de RRHH.

Tal y como destaca Xifra (2011), los objetivos sociales y económicos de una empresa no son excluyentes, por lo que adoptar decisiones para fomentar la felicidad y el bienestar de los empleados beneficia tanto a los públicos internos 
como a la propia organización. Y es que las razones esgrimidas para fomentar la felicidad dentro de las organizaciones superan los meros planteamientos éticos. Los efectos beneficiosos para la cuenta de resultados que se desprenden del fomento de la felicidad en forma de aumento de productividad o retención del talento también le otorgan a esta tendencia una motivación instrumental.

Pese a todo, en España, es una función que no se aborda de manera generalizada en las empresas, y mucho menos de forma estructurada y planificada. Es común que las organizaciones adopten algunas acciones para mejorar su clima interno y fidelizar a sus trabajadores, pero en general adolecen de estructuras organizativas que permitan un desarrollo integral de estas medidas. Entre las empresas que sí gestionan de modo formal la felicidad y el bienestar de sus empleados existe una gran heterogeneidad en cuanto a la asignación de responsabilidades e incluso en la nomenclatura de las estructuras y secciones de las que dependen. Esto indica que se trata de un campo aún en desarrollo y que cada entidad interpreta conforme a su organigrama, normas y valores, por lo que su conceptualización no se encuentra unificada. Sin embargo, en comparación con su escasa implementación en el tejido empresarial, sí existe un conocimiento por parte de los mandos tanto del puesto de director de Felicidad o Chief happiness officer como de sus funciones, probablemente por influencia de tendencias empresariales provenientes de otros países.

Los resultados de esta investigación son indicativos de buenas prácticas desarrolladas en la actualidad en el tejido empresarial español, ya que la muestra está formada por organizaciones previamente reconocidas por su buen clima laboral, comunicación y cuidado de su plantilla. Sus limitaciones se concretan en que no son generalizables a todas las empresas nacionales ya que el área de estudio, como es la relación entre la $\mathrm{Cl}$ y la felicidad y el bienestar de los empleados, se encuentra en un estado incipiente en España y aún existen muchas entidades que no ahondan en estos aspectos.

De cara a futuras investigaciones sería relevante continuar profundizando en el modo en que las empresas abordan la felicidad de sus plantillas y las consecuencias que esto puede tener sobre aspectos como la productividad o la retención del talento para conocer los aportes con los que puede contribuir la $\mathrm{Cl}$ a estas áreas de gestión organizativa.

\section{Referencias}

Aced, Cristina (2013). Relaciones públicas 2.0. Cómo gestionar la comunicación corporativa en el entorno digital. Barcelona: Editorial UOC. ISBN: 9788490297940

Aguadero-Fernández, Francisco (2013). Relaciones públicas y comunicación. Un enfoque estratégico. México: LID Editorial Mexicana. ISBN: 9786077610892

Almansa-Martínez, Ana (2005). "Relaciones públicas y gabinetes de comunicación". Anàlisi: quaderns de comunicació $i$ cultura, n. 32, pp. 117-132.

https://www.raco.cat/index.php/analisi/article/viewFile/15175/179895

Almansa-Martínez, Ana (2011). Del gabinete de prensa al gabinete de comunicación: la dirección de comunicación en la actualidad, Zamora: Comunicación Social.

Andrade, Horacio (2005). Comunicación organizacional interna: proceso, disciplina y técnica. La Coruña: Netbiblo.

Argenti, Paul A. (2014). Comunicación estratégica y su contribución a la reputación. Madrid: LID editorial.

Arribas-Urrutia, Amaia (2000). "Comunicación en la empresa. La importancia de la información interna en la empresa”. Revista latina de comunicación social, v. 3, n. 27.

http://www.revistalatinacs.org/aa2000tma/127amaia.html

Barbeito-Veloso, María-Luz; Perona-Páez, Juan-José (2019). “Comunicación organizacional en clave sonora: el caso de Blink, los podcasts de BBVA". El profesional de la información, v. 28, n. 5, e280511.

https://doi.org/10.3145/epi.2019.sep.11

Bardon, Thibaut; Josserand, Emmanuel (2018). “Management innovations from a Foucauldian perspective: Time to take action".M@n@gement, v. 21,n.4,pp.1244-1263.

https://www.cairn.info/revue-management-2018-4-page-1244.htm

Barel, Yvan; Frémeaux, Sandrine (2016). “Bonheur au travail: les trois conditions de la réussite”. Gestion, v. 41, n. 2, pp. 82-84.

https://www.cairn.info/revue-gestion-2016-2-page-82.htm

Barquero, José-Daniel; Pérez-Senac, Román; Barquero, Mario (2010). Dirección estratégica de relaciones públicas. Barcelona: Profit Editorial. ISBN: 9788492956036

Barranco, Francisco-Javier (1993). Planificación estratégica de recursos humanos, del marketing interno a la planificación. Madrid: Ediciones Pirámide. ISBN: 9788436807714 
Bauer, Caroline (2017). "Work and happiness in Calvin's thought". Revue d'histoire de la pensée économique. Classiques Garnier, 2017-2, n. 4, pp. 75-96.

https://doi.org/10.15122/isbn.978-2-406-07355-0.p.0075

Beau, Pauline (2019). “Un nouveau gestionnaire: le «responsable du bonheur»”. Gestion, v. 44, n. 2, pp. $34-37$.

https://www.cairn.info/revue-gestion-2019-2-page-34.htm

Berceruelo, Benito (coord.) (2011). Comunicación interna en la empresa. Claves y desafíos. Vizcaya: Aedipe. ISBN: 978 8487670985

Boniwell, Ilona; Chabanne, Justine (2017). “La psychologie positive appliquée au travail”. Le journal des psychologues, 2017/4, n. 346, pp. 33-36.

https://doi.org/10.3917/jdp.346.0033

Carrillo-Durán, María-Victoria (2016). “Importancia del profesional de la información en la dirección de comunicación de las organizaciones". El profesional de la información, v. 25, n. 2, pp. 272-278.

https://doi.org/10.3145/epi.2016.mar.14

Castillo-Esparcia, Antonio (2008). Introducción a las relaciones públicas. Málaga: Instituto de Investigación en Relaciones Públicas (IIRP).

https://dialnet.unirioja.es/servlet/articulo?codigo $=4851611$

Castro-Martínez, Andrea; Díaz-Morilla, Pablo (2019). "Análisis del ranking Great Place to Work y de los premios del Observatorio de Comunicación Interna: prácticas de comunicación interna en empresas españolas (2014-2018)". El profesional de la información, v. 28, n. 5, e280518.

https://doi.org/10.3145/epi.2019.sep.18

Cebrián-Herreros, Mariano (2012). Periodismo empresarial e institucional. Zamora: Comunicación Social. ISBN: 97884 15544081

Cuenca-Fontbona, Joan; Verazzi, Laura (2018). Guía fundamental de la comunicación interna. Barcelona: Editorial UOC. ISBN: 9788491802723

Cutlip, Scott M.; Center, Allen H.; Broom, Glen M. (2001). Relaciones públicas eficaces. Barcelona: Gestión 2000. ISBN: 9788480885737

D’Almeida, Nicole; Libaert, Thierry (2018). La communication interne des entreprises. Malakoff: Dunod, 8a éd. ISBN: 978 2100774845

Dasgupta, Shilpee A.; Suar, Damodar; Singh, Seema (2013). “Impact of managerial communication styles on employees' attitudes and behaviours". Employee relations, v. 35, n. 2, pp. 173-199.

https://doi.org/10.1108/01425451311287862

Delassus, Éric (2019). “Le bonheur au travail: un exemple de mauvaise foi managériale?”. Septième congrès de la Société de Philosophie des Sciences de Gestion (SPSG), May 2019. Paris: France.

https://hal.archives-ouvertes.fr/hal-02133761

De-las-Heras-Pedrosa, Carlos; Ruiz-Mora, Isabel; Paniagua-Rojano, Francisco-Javier (2018). Gestión de la comunicación en las instituciones. Madrid: Pearson. ISBN: 9781787268616

Del-Pozo-Lite, Marisa (1997). Cultura empresarial y comunicación interna. Su influencia en la gestión estratégica. Madrid: Editorial Fragua. ISBN: 9788470740954

Del-Pozo-Lite, Marisa (2000). Gestión de la comunicación interna en las organizaciones. Casos de empresa. Navarra: Ediciones Universidad de Navarra-Eunsa. ISBN: 9788431318253

Díaz-Soloaga, Paloma (2019). "The role of communication in organizational culture. Is there a pattern in Spanish fashion companies?". El profesional de la información, v. 28, n. 5, e280506.

https://doi.org/10.3145/epi.2019.sep.06

Fall, Amar; Safy-Godineau, Fatéma; Carassus, David (2018). "Perceptions de justice organisationnelle dans les collectivités locales: quels impacts sur le bien-être psychologique au travail et sur l'intention de quitter des agents?”. @GRH, De Boeck Supérieur, 2018/4, n. 29, pp. 31-59.

https://doi.org/10.3917/grh.184.0031

Feuvrier, Marie-Pierre (2014). “Bonheur et travail, oxymore ou piste de management stratégique de l'entreprise?". Management \& avenir, 2014/2, n. 68, pp. 164-182.

https://www.cairn.info/revue-management-et-avenir-2014-2-page-164.htm

Frayne, David (2018). Le refus du travail. Théorie et pratique de la résistance au travail. Paris: Éditions du Détour. ISBN: 9791097079352 
Freeman, R. Edward; Reed, David L. (1983). "Stockholders and stakeholders: A new perspective on corporate governance". California management review, v. 25, n. 3, pp. 88-106.

https://bit.ly/2Xqvvbx

Frey, Bruno S. (2018). Economics of happiness. Switzerland: Springer.. ISBN: 9783319758077

https://doi.org/10.1007/978-3-319-75807-7_11

García-Uceda, Esperanza (2016). “Gestión estratégica de la comunicación interna. Un caso de red informal en una organización educativa”. Opción, v. 32, n. especial 7, pp. 684-706.

https://produccioncientificaluz.org/index.php/opcion/article/view/21501

Grunig, James E.; Hunt, Todd (2003). Dirección de relaciones públicas. Barcelona: Gestión 2000. ISBN: 9788480889483

Guillemin, Michel (2018). “Le bonheur au travail: qu'en disent les scientifiques?”. Environnement, risques \& santé, v. 17, n. 5, pp. 487-497.

https://doi.org/10.1684/ers.2018.1218

Hassani, Nadia (2017). "Chief happiness officers: les nouvelles technologies de l'information et de la communication au service du bonheur au travail". Communication management, v. 14, n. 2, pp. 99-114.

https://www.cairn.inforevue-communication-et-management-2017-2-page-99.htm

Huang, Jin; Yang, Aimei (2015). "Implementing dialogic communication: A survey of IPR, PRSA, and IABC members". Public relations review, v. 41, n. 3, pp. 376-377.

https://doi.org/10.1016/j.pubrev.2015.02.003

IFOP (2020). Bonheur, sens du travail et raison d'être: le regard des salariés français sur l'entreprise. Paris: Institut d'études opinion et marketing en France et à l'international.

https://www.ifop.com/wp-content/uploads/2020/01/117022-pr\%C3\%A9sentation-pour-publication.pdf

Karanges, Emma; Johnston, Kim; Beatson, Amanda; Lings, lan (2015). "The influence of internal communication on employee engagement: A pilot study". Public relations review, v. 41, n. 1, pp. 129-131.

https://doi.org/10.1016/j.pubrev.2014.12.003

Masrouki, Syrine (2019). “Les tiers-lieux en tant qu'espaces de travail: effet de l'élément naturel sur le bien-être au travail". Journée doctorale SHS du Grand-Est, Apr 2019, Nancy, France.

https://halshs.archives-ouvertes.fr/halshs-02323766

Medina-Aguerrebere, Pablo (2012). “El valor estratégico de la comunicación”. Revista de comunicación y salud, v. 2, n. 1, pp. 19-28.

https://doi.org/10.35669/revistadecomunicacionysalud.2012.2(1).19-28

Miquel-Segarra, Susana; Aced, Cristina (2019). "El perfil de los responsables de comunicación interna en España”. Revista de la Asociación Española de Investigación de la Comunicación, v. 6, n. 11, pp. 99-118.

http://revistaeic.eu/index.php/raeic/article/view/184

Najeh, Hajer (2019). "The function "Chief happiness officer" and the double performance. Reality and perspectives in African countries. Case of B2S Morocco". Journal of behavior studies in organizations, n. 2, pp. 18-29.

https://doi.org/10.32038/JBSO.2019.02.03

Porath, Christine; Spreitzer, Gretchen (2016). "Créer une performance durable". Harvard business review, décembre 2015-janvier 2016.

https://www.hbrfrance.fr/magazine/2015/11/8741-creer-une-performance-durable

Ramilo-Méndez, Nicolás (2012). "10 años de Great Place to Work en España". Capital humano: revista para la integración y desarrollo de los recursos humanos, n. 25, n. 266, pp. 54-59.

Robertson, Ivan; Cooper, Cary (2011). Well-being: Productivity and happiness at work. Palgrave Macmillan. ISBN: 9780 230306738

Rojas-Orduña, Octavio-Isaac (2012) Relaciones públicas. La eficacia de la influencia. 3a ed. Madrid: Editorial ESIC. ISBN: 9788473568777

Ulloa-Tapia, César-Alfonso; Apolo-Buenaño, Diego-Eduardo; Villalobos-Arqueros, Juan-Alejandro (2015). "Aproximación conceptual a la comunicación corporativa: retos y propuestas". Austral comunicación, v. 4, n. 2, pp. $287-301$. https://ojs.austral.edu.ar/index.php/australcomunicacion/article/view/137

Vanhée, Laurence (2013). Happy RH: Le bonheur au travail, rentable et durable. La Charte, Professional Publishing. ISBN: 2874033146

Vasconcelos, Anselmo-Ferreira (2008). "Broadening even more the internal marketing concept". European journal of marketing, v. 42 n. 11/12, pp. 1246-1264. 
https://doi.org/10.1108/03090560810903664

Vázquez-Sande, Pablo; García-Abad, Lito; Pineda-Martínez, Paula (2019). "Comunicación interna y crisis reputacional. El caso de la Universidad Rey Juan Carlos". Revista latina de comunicación social, n. 74, pp. 1748-1760.

http://www.revistalatinacs.org/074paper/1408/91es.html

Vilanova-Giralt, Núria (2016). Micropoderes. Comunicación interna para empresas con futuro. 4a ed. Barcelona: Plataforma Editorial. ISBN: 9788415750673

Warr, Peter (2013). "Fuentes de felicidad e infelicidad en el trabajo: una perspectiva combinada". Revista de psicología del trabajo y de las organizaciones, v. 29, n. 3, pp. 99-106.

https://doi.org/10.5093/tr2013a15

Wimmer, Roger D.; Dominick, Joseph R. (1996). La investigación científica de los medios de comunicación: una introducción a sus métodos. Barcelona: Bosch. ISBN: 847676359 X

Xifra, Jordi (2005). Planificación estratégica de relaciones públicas. Barcelona: Paidós Ibérica. ISBN: 9788449317781

Xifra, Jordi; Lalueza, Ferrán (2009). Casos de relaciones públicas y comunicación corporativa. Madrid: Pearsons. ISBN: 8483226111

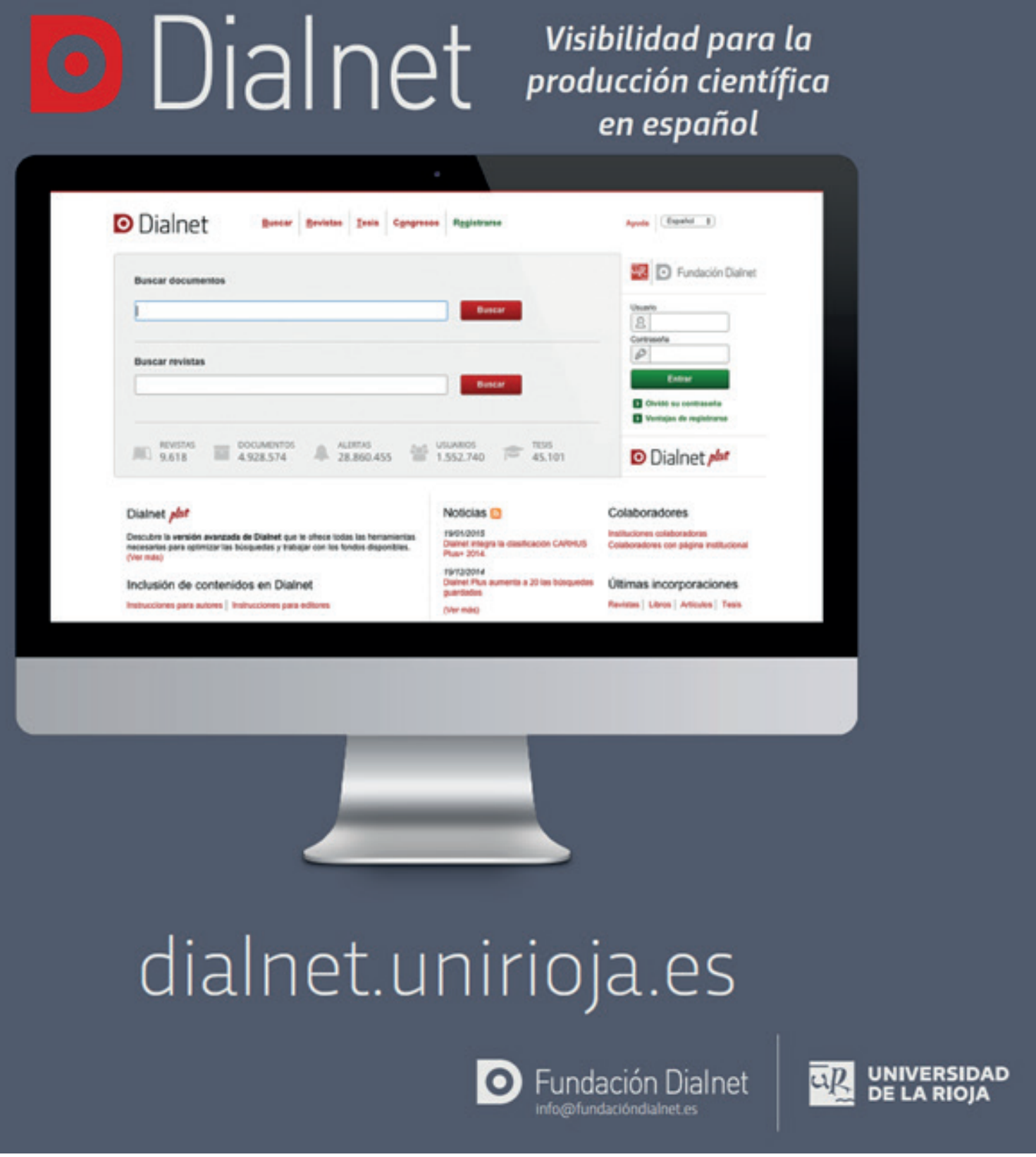

